\title{
TCR cross-reactivity and allorecognition: new insights into the immunogenetics of allorecognition
}

\author{
L. J. D'Orsogna • D. L. Roelen • I. I. N. Doxiadis • \\ F. H. J. Claas
}

Received: 4 September 2011 / Accepted: 11 November 2011 /Published online: 3 December 2011

(C) The Author(s) 2011. This article is published with open access at Springerlink.com

\begin{abstract}
Alloreactive $\mathrm{T}$ cells are core mediators of graft rejection and are a potent barrier to transplantation tolerance. It was previously unclear how $\mathrm{T}$ cells educated in the recipient thymus could recognize allogeneic HLA molecules. Recently it was shown that both naïve and memory $\mathrm{CD} 4^{+}$and $\mathrm{CD} 8^{+} \mathrm{T}$ cells are frequently cross-reactive against allogeneic HLA molecules and that this allorecognition exhibits exquisite peptide and HLA specificity and is dependent on both public and private specificities of the $\mathrm{T}$ cell receptor. In this review we highlight new insights gained into the immunogenetics of allorecognition, with particular emphasis on how viral infection and vaccination may specifically activate alloHLA reactive $T$ cells. We also briefly discuss the potential for virus-specific $\mathrm{T}$ cell infusions to produce GvHD. The progress made in understanding the molecular basis of allograft rejection will hopefully be translated into improved allograft function and/or survival, and eventually tolerance induction.
\end{abstract}

Keywords Allorecognition · Memory T cells $\cdot$ Antiviral immunity · Heterologous immunity . Tissue specificity. Alloantibodies

L. J. D’Orsogna $(\bowtie) \cdot$ D. L. Roelen · I. I. N. Doxiadis •

F. H. J. Claas

Department of Immunohematology and Blood Transfusion,

Leiden University Medical Centre,

PO Box 9600, 2300RC, Leiden, the Netherlands

e-mail: lloyd.dorsogna@health.wa.gov.au

\section{J. D’Orsogna}

University of Western Australia,

Perth, WA, Australia

L. J. D’Orsogna

PathWest Laboratory Medicine,

Nedlands, WA, Australia
Thymic educated naïve $T$ cells are inherently cross-reactive and commonly allo-HLA reactive

The first phase of $\mathrm{T}$ cell development is the production of a functional $\mathrm{T}$ cell receptor (TCR), irrespective of antigen specificity. Newly rearranged TCR repertoire must be capable of recognizing all possible HLA molecules as the $T$ cell repertoire is generated before encountering the actual autologous HLA molecules present in the thymus. Zerrahn and colleagues studied MHC reactivity of thymocytes in MHC class I and II-deficient mice (Zerrahn et al. 1997). A limiting dilution analysis of $\mathrm{CD}^{+}$thymocytes was employed as a means to assess MHC reactivity of the selected thymocytes. MHC reactivity in the preselection repertoire was very high, but no higher than in the normal TCR repertoire. Cross-reactivity of clones with multiple MHC molecules occurred to a similar extent in the preselection and MHC-selected repertoires. T cells reacted promiscuously with several or many $\mathrm{MHC}$ molecules with $\mathrm{T}$ cell clones from MHC class I and II-deficient mice reacting with up to three or four of the eight tested $\mathrm{H}-2$ haplotypes. These results established the high level and promiscuity of allogeneic MHC reactivity in the germline $\mathrm{T}$ cell repertoire prior to positive and negative selection.

The TCR repertoire that actually exits the thymus is then the product of "positive" and "negative" selection based on self-peptide/autologous HLA recognition in the thymus. Any T cells having receptors that respond with high affinity to complexes of self-peptide and autologous MHC class I or II molecules are eliminated, a process termed negative selection. However this quality control mechanism encompasses only HLA isoforms expressed by that individual (autologous HLA), and not by other HLA isoforms (allogeneic HLA) (Greisemer et al. 2010; Marrack and Kappler 1988; Schild et al. 1990). Accordingly T cells that 
can respond to complexes of self-peptide and allogeneic HLA class I and II molecules are able to exit the thymus as they are not negatively selected and can be positively selected by their natural high level of cross-reactivity (Bankovich and Garcia 2003; Borbulevych et al. 2009; Colf et al. 2007; Borst et al. 1987; Ely et al. 2008; Marrack and Kappler 1988). T cells that have survived positive and negative selection leave the thymus and enter the circulation as mature naïve T cells. Allogeneic HLA molecules with even a single amino acid substitution as compared to autologous HLA molecules can cause strong alloreactivity in vivo (Fleischhauer et al. 1994; Herman et al. 1999). Mature naïve $\mathrm{T}$ cells exhibit a high frequency $(10 \%)$ of cross-reactivity against mismatched allogeneic HLA molecules from one individual to which they have not been previously exposed (Zerrahn et al. 1997; Macedo et al. 2009; Golshayan et al. 2010).

The role of the thymus in tolerance to specific alloantigens

The rearrangement of TCR germ line DNA sequences and the pairing of the $\alpha$ and $\beta$ TCR molecules create a theoretical repertoire diversity of about $10^{15}$ different $\mathrm{T}$ cells in humans. Each of these newly generated TCRs has a very high level of inherent specific cross-reactivity (Selin and Brehm 2007; Mason 1998), including allogeneic HLA cross-reactivity.

Tissue-specific proteins are expressed in the thymus and $\mathrm{T}$ cells that bind self-peptides presented on self-HLA molecules are removed in the thymus by negative selection. For example, the EBV EBNA3A-specific TCR with TRVB6 gene segment usage is specific for the EBV FLRGRAYGL peptide presented by HLA-B*08:01 (Burrows et al. 1994; Argaet et al. 1994). This TCR also binds the EEYLQAFTY selfpeptide from the $\mathrm{ABCD} 3$ gene presented on HLA-B*44:02 (Macdonald et al. 2009). In HLA-B8 B44 heterozygous individuals, this TCR is negatively selected in the thymus to avoid autoimmunity (Burrows et al. 1995), but in HLA-B $8^{+} \mathrm{B} 44^{-}$individuals, this $\mathrm{T}$ cell is positively selected and enters the circulation as a naïve $T$ cell which can mount an immune response against EBV in the setting of EBV infection and potentially against allo-HLA-B*44:02 molecules in the transplantation setting.

Structural studies show that this single EBV EBNA3Aspecific TCR with Vb6 usage specifically recognizes both the natural viral ligand on the HLA-B8 molecule and the alloantigen HLA-B*44:02, in both peptide and HLAspecific fashion (Gras et al. 2009; Macdonald et al. 2009). In HLA-B8 B44 heterozygote individuals, the TCR which recognizes the FLR peptide on HLA-B8 employs an entirely different $\mathrm{Vb}$ segment usage and mode of TCR docking, which coincides with a polymorphic region between HLA-B8 and HLA-B44:02 (Gras et al. 2009).
The markedly contrasting footprints of the two TCRs which both bind to the same viral peptide on HLA-B8 provide a portrait of how self-tolerance shapes the $\mathrm{T}$ cell clonotypes selected into the immune repertoire.

Therefore the $\mathrm{T}$ cell receptor repertoire for a viral epitope is diversified by tolerance to background autologous major histocompatibility complex antigens (Burrows et al. 1995; Gras et al. 2009), but not alloantigens.

Naïve T cells and primary alloresponses

$\mathrm{T}$ cell alloimmunity is of extraordinary potency, up to $10 \%$ of recipient $\mathrm{T}$ cells may react to donor alloantigens; in contrast only a very small proportion of $\mathrm{T}$ cells respond to any given pathogen-related epitope. While the dominant role of $\mathrm{T}$ cell alloimmunity in allograft rejection was previously known, the precise contribution of naïve and memory $\mathrm{T}$ cells was only recently clarified. Macedo and colleagues used a flow cytometry-based method to analyze alloreactivity from human $\mathrm{T}$ cell subsets. $\mathrm{CD} 4^{+}$and $\mathrm{CD} 8^{+} \mathrm{T}$ cells proliferate at comparable levels and contain similar alloreactive precursor frequencies (Macedo et al. 2009). Furthermore naïve and memory $\mathrm{T}$ cells proliferate equally when stimulated with allogeneic cells in vitro. Proliferation was alloantigen specific. Similar findings are reported for direct allorecognition by naïve versus memory $\mathrm{T}$ cells in mice (Golshayan et al. 2010).

The high precursor frequency of alloreactive $\mathrm{T}$ cells is likely the result of the inherent bias of prethymic repertoire towards MHC recognition and the high level of crossreactivity inherent to all TCRs (Webb and Sprent 1986).

\section{Memory $\mathbf{T}$ cells in primary alloresponses}

Alloreactive memory $\mathrm{T}$ cells can be generated by previous transplantation, blood transfusion, pregnancy, or viral infection. $\mathrm{T}$ cell alloresponses against HLA antigens to which the recipient has already been exposed have already been extensively studied and the immunological mechanisms of the alloreactivity are understood. However it was previously unclear why even non-sensitized recipients have a very high proportion of preexisting alloreactive memory $\mathrm{T}$ cells. Recent studies reveal that the explanation for the presence of alloreactive memory $\mathrm{T}$ cells in non-sensitized recipients is likely cross-reactive from pathogen-specific memory $\mathrm{T}$ cells generated as a result of previous immunological (pathogen) exposure (Amir et al. 2010).

While it has been shown that $\mathrm{T}$ cells with alloreactive potential exist equally within the naïve and memory $\mathrm{T}$ cell subsets in vitro, it is more likely that memory $\mathrm{T}$ cell subsets are responsible for clinical organ rejection (Welsh et al. 2000; Welsh and Selin 2002; Adams et al. 2003; Brook et 
al. 2006). Memory $\mathrm{T}$ cells have lower activation requirements and less need for co-stimulation, and are more likely to express molecules with cytotoxic function (Flynn and Mullbacher 1996; Burrows et al. 1999; Hamann et al. 1997; Byrne et al. 1988). Furthermore memory T cells are more likely a home to sites of active inflammation while naïve $\mathrm{T}$ cells remain primarily within central lymphoid organs. Certain immunosuppressive drugs are also less effective at suppressing memory $\mathrm{T}$ cell responses, versus naïve $\mathrm{T}$ cell responses (Pearl et al. 2005).

\section{Viral-specific memory T cells}

Allo-HLA cross-reactivity by preexisting viral-specific memory $\mathrm{T}$ cells explains the presence of alloreactive memory $\mathrm{T}$ cells in non-sensitized individuals (Amir et al. 2010; Yang and Welsh 1986; Yi-qun et al. 1996; Wang et al. 2008; Welsh and Selin 2002). Alloreactivity by viral-specific memory T cells is common (Amir et al. 2010). We recently showed that $45 \%$ of viral-specific memory $\mathrm{T}$ cells specifically cross-reacted against at least one allogeneic HLA molecule (see Fig. 1). Allo-HLA cross-reactivity was shown for both HLA class I and II molecules, and surprisingly two viral-specific $\mathrm{CD} 8^{+} \mathrm{T}$ cells were shown to cross-react against allogeneic HLA class II molecules, as was also shown by others (Rist et al. 2009). These allo-HLA cross-reactive responses also showed tissue specificity depending on presentation of tissue-specific selfpeptides (Amir et al. 2010, 2009, D’Orsogna et al. 2011a, b, c). An inventory of human viral-specific memory $\mathrm{T}$ cells reported to give specific allo-HLA cross-reactivity has recently been published (D’Orsogna et al. 2010).
Antiviral $\mathrm{T}$ cell responses in individuals with similar HLA molecules share similarities in epitope hierarchies and $\mathrm{T}$ cell receptor variable gene usage (public specificities) (Venturi et al. 2008), yet even with the same Vb usage, the $\mathrm{T}$ cell receptor amino acid sequences can differ between individuals (private specificities). Both public and private specificities of viral-specific memory $\mathrm{T}$ cells are important to the field of allorecognition and organ rejection as they give rise to different patterns of allo-HLA cross-reactivity, which are generally not predictable.

\section{Public and nonpublic TCR responses}

EBV infection in a HLA-B*08:01 individual gives rise to a public TCR response recognizing the EBV peptide FLRGRAYGL presented on HLA-B*08:01. Burrows and colleagues demonstrated that the HLA-B8/FLR restricted response always gives rise to a public BV6S2 TCR which cross-reacts against allogeneic HLA-B*44:02, but not HLA-B*44:03. This finding has been reproduced independently by multiple groups (Burrows et al. 1994; D’Orsogna et al. 2009; Macedo et al. 2009; Gaston et al. 1983). Other public viral-specific $T$ cell immune responses have been reported (Venturi et al. 2008); however, alloHLA cross-reactivity from these public TCRs has not been studied, but could also give rise to predictable allo-HLA cross-reactivity.

Nonetheless most viral-specific T cell responses do not give rise to a public TCR or predictable allo-HLA crossreactivity. For example, variable allo-HLA cross-reactivity by $\mathrm{T}$ cell clones sorted from the same individual with the

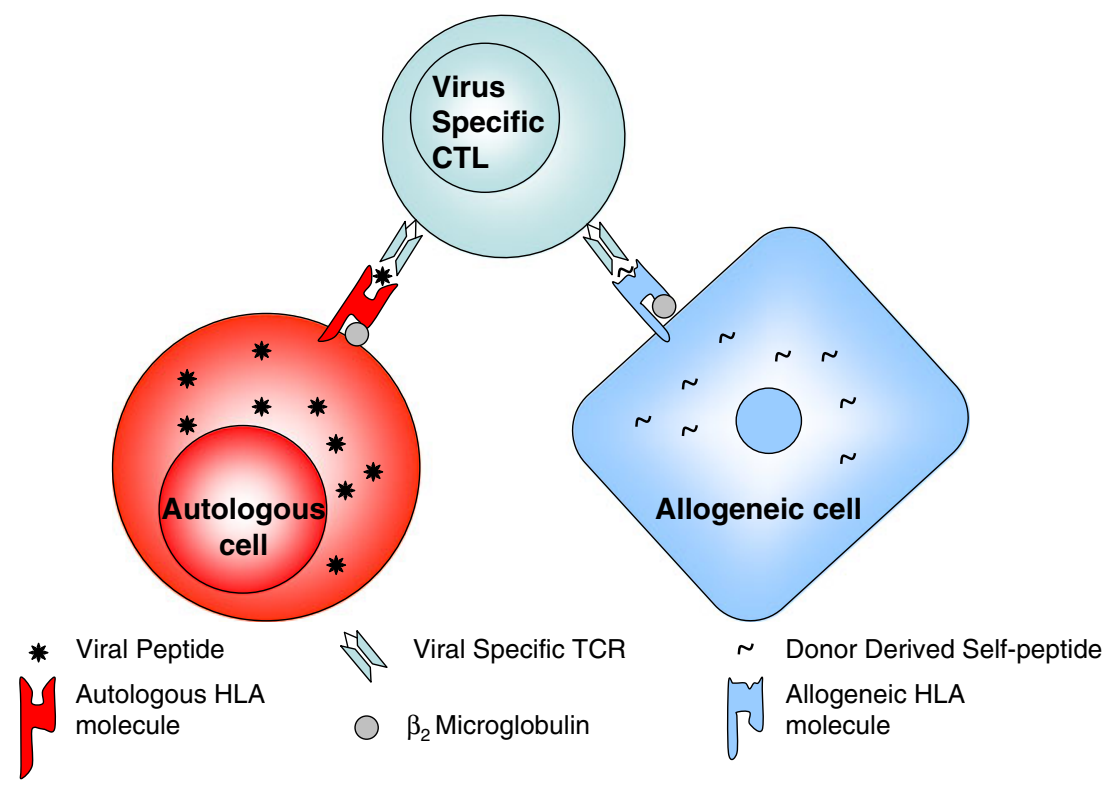

Fig. 1 Allo-HLA cross-reactivity by viral-specific memory $\mathrm{T}$ cells. Viral-specific memory $\mathrm{T}$ cells target virus-infected autologous cells presenting viral peptides in a self-HLA restricted fashion. The same viral-specific TCR may cross-react against an allogeneic HLA molecule presenting a self-peptide. CTL cytotoxic T lymphocyte. (Figure reproduced with permission from D'Orsogna et al. 2010) 
same specificity, but different TCR $\mathrm{Vb}$ usage, has been reported (Amir et al. 2010). Single cell sorting of T cells specific for the IE62 protein of VZV from an individual with VZV infection generated three different clones using $\mathrm{Vb} 21.3$, Vb14, and an undetermined $\mathrm{Vb}$, which were crossreactive against allogeneic HLA-A*02:05, HLA-B*55:01, and HLA-B*57:01, respectively. Viral-specific memory $T$ cells with the same specificity but different TCR Vb usage clearly give different patterns of allo-HLA cross-reactivity (Amir et al. 2010; Burrows et al. 1997).

Therefore allo-HLA cross-reactivity cannot be predicted based on viral (environmental) antigen exposure and HLA mismatching of the donor and recipient alone. Given the enormous amount of memory $\mathrm{T}$ cell diversity generated as part of a normal immune response, it is likely that a single viral infection may generate memory $\mathrm{T}$ cells with crossreactivity against many different allo-HLA molecules, thus accounting for the lack of association between given viral environmental exposures and unacceptable HLA mismatches. We therefore suggest that approaches aiming to induce tolerance are most likely to be successful when pursued on an individual (autologous) basis, as reported by others (Francis et al. 2011; Jiang et al. 2006; Nagahama et al. 2007; Feng et al. 2009).

\section{Private specificities}

The specific allo-HLA cross-reactivity of a viral-specific memory $\mathrm{T}$ cell is dependent on the specific viral peptide recognized, the HLA restriction element, and the TCR Vb usage of the memory T cell (Amir et al. 2010). However alloHLA cross-reactivity may also be associated with private specificities of the TCR. For example, an influenza IMPspecific $\mathrm{T}$ cell clone (HLA-A2/GIL restricted) with TCR Vb17 usage cross-reacted against allogeneic HLA-B*64:01 (Amir et al. 2010). However another clone with the same specificity generated from the same individual and with the same TCR Vb usage did not recognize allogeneic HLAB*64:01. These two clones contained private specificities within the CDR3 region of the TCR which was presumably responsible for the differing alloreactivity. A similar finding was also reported for two CMV pp65-specific T cell clones (HLA-B7/RPH restricted) from the same individual and with the same TCR Vb usage (Amir et al. 2010).

Brehm and colleagues confirmed that allografts stimulate cross-reactive virus-specific memory $\mathrm{CD} 8 \mathrm{~T}$ cells with private specificity, in an in vivo mouse transplant model (Brehm et al. 2010). Cross-reactive CD8 LCMV-specific memory $\mathrm{T}$ cells proliferated in vivo in response to allografts. CD8 T cells specific to several LCMV epitopes proliferated in response to alloantigens, with the magnitude and hierarchy of epitope-specific responses differing with the private specificities of the host memory $\mathrm{T}$ cell repertoire. Importantly they also showed that purified LCMV-specific CD8 $\mathrm{T}$ cells rejected skin allografts in SCID mice using adoptive transfer studies, even in the absence of B cells, CD4 T cells, and APCs.

Thus viral-specific memory $\mathrm{T}$ cells generated within or between individuals with the same viral antigen specificity and TCR $\mathrm{Vb}$ usage can also give differing patterns of alloHLA cross-reactivity, depending on single amino acid differences within the CDR3 of the TCR (Brehm et al. 2010; Amir et al. 2010). For this reason allo-HLA cross-reactivity by viral-specific memory $\mathrm{T}$ cells can often not be predicted.

\section{Vaccination-induced alloreactivity}

Transplant candidates and recipients are at increased risk of infectious complications and therefore current guidelines recommend that transplant candidates complete the full complement of recommended vaccinations prior to transplantation (Anonymous 2004). However recent evidence suggests that not only naturally acquired infections but also vaccinations can augment the alloreactive $T$ cell repertoire.

Danziger-Isakov and colleagues analyzed the humoral and cellular reactivity in subjects undergoing influenza vaccination (Danziger-Isakov et al. 2010). Serial blood samples from healthy subjects and transplant recipients who received influenza vaccination were taken before and after vaccination and alloreactive T cell immunity was measured, using IFN $-\gamma$ ELISPOT. Vaccination specifically and significantly augmented cellular immunity against a panel of allogeneic stimulators in transplant recipients within 4 weeks of vaccination. These findings demonstrate that influenza vaccination can have a significant impact on the potency of the alloreactive $\mathrm{T}$ cell repertoire, although the underlying immunological mechanism behind the vaccine-induced alloreactivity and its HLA specificity was not determined.

Recent work by our group suggests that specific alloHLA cross-reactivity from de novo vaccine-induced memory $\mathrm{T}$ cells may underlie the increased alloreactivity observed in the previous study. VZV-specific $T$ cells generated de novo after varicella vaccination were cloned and tested for alloreactivity against a panel of allogeneic cells and proximal tubular epithelial cells present in kidney grafts (D’Orsogna et al. 2011a, b, c). A VZV-specific CD8 ${ }^{+}$ memory $T$ cell (HLA-A2 restricted) generated de novo after varicella vaccination of a kidney transplantation candidate specifically cross-reacted against the allogeneic HLAB*55:01 molecule only. This VZV vaccine-induced memory $\mathrm{T}$ cell exhibited cytotoxic effector function, not just cytokine production, against a human HLA-B*55:01 expressing proximal tubular epithelial cell line thus confirming the relevance to clinical kidney transplantation. Therefore vaccination, and not just naturally acquired viral infection, can induce allo-HLA reactive memory $\mathrm{T}$ cells in 
non-sensitized individuals (D’Orsogna et al. 2011a, b, c). Vaccination may also induce de novo $\mathrm{CD}^{+}$memory $\mathrm{T}$ cells with specific allogeneic HLA class II cross-reactivity (Umetsu et al. 1985).

\section{The expression of tissue-specific self-peptide is required for allorecognition}

The recognition of allogeneic HLA molecules by memory $\mathrm{T}$ cells is dependent on presentation of selfpeptide by the allogeneic target cell (Archbold et al. 2006) (see Fig. 1). Our group studied the extensively described cross-reactivity of the EBV EBNA3A T cell against allogeneic HLA-B*44:02 (D’Orsogna et al. 2011a, $\mathrm{b}, \mathrm{c})$, as this is the only human viral-specific memory $\mathrm{T}$ cell for which an allopeptide (EEYLQAFTY from ABCD3 gene) has been reported (Macdonald et al. 2009). Allogeneic HLA-B*44:02 proximal tubular epithelial cells and human umbilical endothelial cells are poor targets for EBV EBNA3A-specific memory $\mathrm{T}$ cells, in contrast to HLA-B*44:02 expressing B cells, PHA blasts, splenocytes, and HLA-B*44:02 transfected K562 cell lines (SALs). HLA-B*44:02 PTECs were specifically lysed by an EBV EBNA3A-specific T cell clone although lysis was low as compared to HLA-B*44:02 PHA blasts and SALs, but lysis was greatly increased by exogenous EEYLQAFTY peptide loading. HLA-B*44:02 HUVECs were not lysed unless exogenously loaded with the EEYLQAFTY peptide. Lack of HLA-B*44:02 expression was excluded as a cause for these results, strongly suggesting that the lack of recognition of HLA-B*44:02 epithelial and endothelial cells by the EBV EBNA3A T cells was due to (tissue specific) lack of EEYLQAFTY peptide presentation (D'Orsogna et al. 2011a, b, c).

Interestingly expression of the $\mathrm{ABCD} 3$ gene within the various HLA-B*44:02 expressing cell lines did not correlate strongly with allorecognition by the EBV EBNA3A-specific $\mathrm{T}$ cell clone. Therefore differences in tissue-specific self-peptide processing or tissue-specific differences in expression of a protein that contains a peptide capable of competing with the EEYLQAFTY peptide for the peptide-binding groove of the HLAB*44:02 molecule could also cause tissue-specific alloreactivity. Alternatively the EEYLQAFTY peptide may not be the natural ligand for the EBV EBNA3A T cells. Regardless, studies using EBV EBNA3A T cells clearly demonstrate that alloreactivity from viral-specific memory $\mathrm{T}$ cells is the result of highly focused, peptide-dependent structural mimicry.

Tissue-specific allorecognition is extensively described in humans and mice. As part of our recent work, allorecognition by viral-specific memory $\mathrm{T}$ cells of normal cell subsets, such as B cells, T cells, PHA blasts, monocytes, dendritic cells, and fibroblasts, was studied (Amir et al. 2010). For example, a CMV pp65-specific T cell clone showed high recognition of HLA-DRB $1 * 08: 01^{+}$ CD40L activated $\mathrm{B}$ cells but low recognition of resting $\mathrm{B}$ cells and fibroblasts. A VZV IE62-specific T cell clone showed high recognition of HLA-B*55:01 dendritic cells and PHA blasts but low recognition of monocytes and $\mathrm{T}$ cells. Lack of HLA expression was excluded in all of these target cells. These results indicate that alloreactivity by human virus-specific memory $\mathrm{T}$ cells against normal cell subsets is frequently cell type specific.

Human tissue-specific alloreactivity is also suggested by studies using graft-infiltrating lymphocytes obtained from renal allografts undergoing rejection (Miltenburg et al. 1989; van der Woude et al. 1990; Deckers et al. 1997, 1998; Yard et al. 1994; Brook et al. 2006). Graft-infiltrating $\mathrm{T}$ cells were shown to exhibit functional activity against proximal tubular cells grown from the corresponding donor, but not donor-derived splenocytes or proximal tubular cells from a third party donor (Miltenburg et al. 1989; van der Woude et al. 1990). In one study $33 \%$ of graft-infiltrating $T$ cell lines reacted in a tissue-specific fashion to donor proximal tubular cells but not donor splenocytes (van der Woude et al. 1990).

Peptide-dependent (tissue-specific) allorecognition is also extensively described in mice models. For example, Molina and colleagues found that the expression of tissuespecific self-peptide is required for allorecognition and Heath and colleagues found that the cell type-specific recognition of allogeneic cells by alloreactive cytotoxic $\mathrm{T}$ cells was a consequence of peptide-dependent allorecognition (Molina and Huber 1990; Heath and Sherman 1991). The immunological mechanisms underlying peptide and HLA-specific TCR cross-reactivity are described elsewhere (Yin and Marluzza 2009; Whitelegg and Barber 2004). Allorecognition can therefore be tissue specific likely because of differential allopeptide presentation which may have important implications for transplantation monitoring and rejection.

\section{Anti-HLA antibodies and heterologous immunity}

The effect of viral infection or vaccination on alloantibody production is not so clear; however, it is theoretically possible that antibodies specific for a viral peptide could cross-react with alloantigens. $\mathrm{T}$ cell help for such an antibody response would simply require a TCR that recognized the same viral peptide (or another peptide from the same viral protein) presented by autologous class II HLA molecules on the $\mathrm{B}$ cell with the cross-reactive antibody specificity. 
A non-allogeneic stimulus can trigger the production of de novo anti-HLA antibodies in healthy adults (Alberu et al. 2007). Hepatitis B vaccination was associated with de novo production of HLA-specific alloantibodies in 9/20 vaccine recipients, but not controls. The alloantibody production resolved within 3 months of vaccination. De novo anti-HLA antibody formation was also reported after pandemic H1N1 and seasonal influenza immunization in kidney transplant recipients (Katerinis et al. 2011). While the immunological mechanisms underlying the alloantibody production were not determined, presumably CD4 T cells recognized a vaccine peptide presented by autologous HLA molecules with homology to allogeneic HLA molecules, thus providing help for an antibody response to the vaccine peptide that was cross-reactive with allogeneic HLA molecules.

Anti-HLA antibodies have also been reported in the sera of non-alloimmunized healthy males and in cord blood (Morales-Buenrostro et al. 2008; El-Awar et al. 2008), and it was suggested that these anti-HLA antibodies are produced to cross-reactive epitopes found in microorganisms, ingested proteins, and allergens making them "natural" antibodies (Morales-Buenrostro et al. 2008). However such antibodies may only recognize denatured forms of HLA or beta-2 microglobulin and may therefore not be clinically relevant in the transplantation setting (Cai et al. 2009; El-Awar et al. 2008). Furthermore, cytotoxic activity of natural donor-specific antibody does not correlate with the antibody level (Cai et al. 2009). The relevance of these natural HLA antibodies to transplantation is therefore not yet known; however, even if they are irrelevant to graft rejection, awareness of these natural anti-HLA antibodies could prove useful in avoiding unnecessary exclusion of potential donors.

Therefore non-allogeneic stimuli can trigger the production of anti-HLA antibodies and some anti-HLA antibodies could actually be "cross-reactive" pathogen-specific antibodies. Further clinical studies are clearly warranted as the longterm clinical significance of anti-HLA antibodies induced following infection or vaccination remains to be addressed.

\section{Discussion}

Adaptive $\mathrm{T}$ cell responses to donor antigens are a potent barrier to successful transplantation. Enormous progress has been made in the understanding of the nature of allorecognition and $\mathrm{T}$ cell-mediated allograft rejection. Thymically educated naïve $\mathrm{T}$ cells are very commonly allo-HLA cross-reactive and can be activated by pathogen exposure to become memory $\mathrm{T}$ cells. Memory $\mathrm{T}$ cells are long lived and have lower activation requirements and less co-stimulation dependence, allowing them to commonly recognize allogeneic cells expressing the allogeneic HLA molecule against which they are crossreactive. This cross-reactivity cannot be predicted based on donor-recipient mismatch and immunological history alone and is dependent on the original cognate viral peptide recognized, the HLA restriction and both the public and private specificities of the TCR.

$\mathrm{T}$ cell alloresponses are thought to occur via two main pathways, direct and indirect allorecognition; however, new understanding of the immunological mechanisms underlying allorecognition means currently used models of $\mathrm{T}$ cell allorecognition may have to be altered. The direct allorecognition pathway was thought to involve the recognition of intact allogeneic MHC:peptide complexes on donor APCs and is thought to only occur early following transplantation when donor APCs are still present in the graft. However recent studies show that both naïve and memory $\mathrm{T}$ cells can cross-react (directly recognize) allogeneic HLA molecules presenting self-peptides on allogeneic tissue cells (see Fig. 1). Memory T cells in particular are able to persist and have lower activation and co-stimulation requirements. We propose that direct recognition of allogeneic HLA molecule:self-peptide complexes on donor cells may be a dominant pathway of $\mathrm{T}$ cell allorecognition and may persist for the life of the graft even after donor APCs are no longer present in the recipient.

Nonetheless direct cross-reactivity of memory $\mathrm{T}$ cells against intact allogeneic HLA molecules presenting a selfpeptide would not result in production of alloantibodies against the same allo-HLA molecule. For example, in our recent report (D'Orsogna et al. 2011a, b, c), there is no immunological mechanism whereby the cross-reactivity of the VZV IE62-specific CD8 T cell against allogeneic HLAB55 could result in the production of an anti-HLA-B55 antibody. Cross-reactivity of viral-specific CD8 T cells against allo-HLA molecules would result in T cell-mediated rejection only.

Indirect allorecognition occurs when a recipient $\mathrm{T}$ cell recognizes peptide fragments of an allogeneic HLA molecule presented on recipient APCs and is thought to be the predominant mechanism underlying chronic $\mathrm{T}$ cell allorecognition. It is undoubted that peptide fragments from allogeneic HLA molecules are presented on autologous HLA molecules; however, a T cell recognizing allo-HLA peptide fragments presented by an autologous HLA molecule would not necessarily be activated to recognize the intact allo-HLA molecule on the surface of allogeneic cells. Such a concept is contrary to the basic immunological principles of $\mathrm{T}$ cell structure and function. $\mathrm{T}$ cells specifically recognize the $3 \mathrm{D}$ structure of a HLA molecule and a short peptide fragment, and if the presenting autologous HLA molecule is not present on the allogeneic cell then that same $\mathrm{T}$ cell could never recognize the 
allogeneic HLA molecule peptide fragment (or the intact allogeneic HLA molecule). However it is highly likely that a CD4 $\mathrm{T}$ cell recognizing allo-HLA peptide fragments presented on recipient APCs (dendritic cells, B cells, or activated $\mathrm{T}$ cells) would stimulate an IgG class switched, high-affinity anti-HLA antibody response from a B cell as part of a germinal center reaction (see Fig. 2).

Therefore recent advances in the understanding of the immunogenetics of allorecognition suggest a new model for allorecognition whereby the majority of $\mathrm{T}$ cell alloresponses may occur via direct recognition (cross-reactivity) by thymically educated naïve and memory $\mathrm{T}$ cells against allogeneic HLA molecules presenting self-peptides (Fig. 1), and indirect recognition may be a major source of anti-HLA antibodies (Fig. 2).

While the authors suggest direct allorecognition by naïve and memory $\mathrm{T}$ cells is the dominant mechanism leading to $\mathrm{T}$ cell-mediated graft rejection, we acknowledge that much work remains to be done. Mifsud and colleagues recently provided the first in vivo quantitative and functional analysis of human cross-reactive virus-specific CD8 T cells in a clinical transplantation framework (Mifsud et al. 2010). EBV EBNA3A-specific $\mathrm{CD} 8^{+} \mathrm{T}$ cells were detectable in the bronchoalveolar lavage fluid of HLA-B*08:01+ recipients who had received a HLA-B*44 mismatched lung allograft. These virus-specific $\mathrm{T}$ cells exhibited a skewed functional phenotype towards cytotoxic but not cytokine-producing capabilities. However the presence of these cells was not associated with poorer clinical outcomes in the absence of EBV reactivation and in the setting of immunosuppression and antiviral prophylaxis. Therefore the clinical relevance of allo-HLA cross-reactive memory $\mathrm{T}$ cells is still unclear, although we suggest viral reactivation would be highly likely to activate alloreactive $\mathrm{T}$ cells in the solid organ transplant setting, as also suggested by others (Selin and Brehm 2007; Welsh and Selin 2002; Adams et al. 2003; Kleim et al. 2008; Gray and Matzinger 1991).

Infusion of virus-specific $\mathrm{T}$ cell lines into bone marrow transplant recipients or immunodeficient patients could also result in graft versus host disease. Melenhorst and colleagues reported that infusion of allogeneic virusspecific $\mathrm{T}$ cells with HLA alloreactivity did not produce GVHD in 73 human subjects (Melenhorst et al. 2010). However the hematopoietic stem cell transplantation recipients in this study received infusions of virus-specific cell lines that were mostly HLA matched, and likely to be fully HLA-A, -B, and -DR matched. Furthermore virus-specific lines were not infused if killing of recipient phytohemagglutinin blasts was greater than $10 \%$ in vitro. We recommend further studies before off the shelf $\mathrm{T}$ cell therapies across HLA barriers enter routine clinical practice (D’Orsogna et al. 2011a, b, c).

Future work should focus on the in vivo relevance of cross-reactivity by viral-specific memory $\mathrm{T}$ cells, in both the solid organ and bone marrow transplantation settings. Allopeptides relevant for human transplantation should be

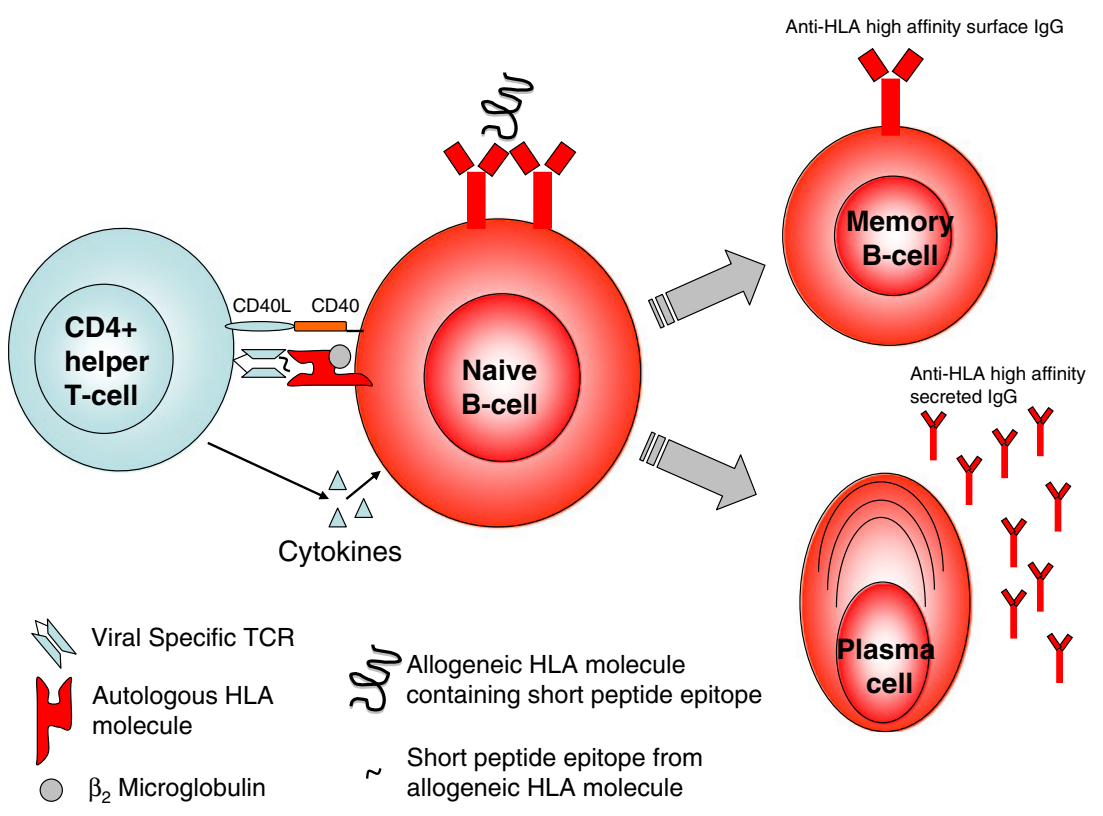

Fig. 2 B cells with specificity for allogeneic HLA must first be activated by the combination of antigen and T cell help. Viral-specific CD4 T cells may cross-react against allogeneic HLA-derived peptides presented on autologous HLA molecules (indirect allorecognition) by recipient APCs (Dendritic cells, B cells, or activated T cells). In the germinal center reaction, such B cells may undergo somatic hypermutation with the development of high-affinity surface $\operatorname{IgG}$ with HLA specificity. Progeny B cells would leave the germinal center and differentiate into either memory B cells or antibody-producing plasma cells. APCs antigen presenting cells 
identified, perhaps commencing with tissue-specific targets of public TCRs. It is clear that pathogen-free mice should be considered a poor model for the induction of transplantation tolerance, considering the important role of viral exposure in the alloimmune response. Clinical research must focus on the effect of viral reactivation, particularly latent herpes viruses, and the benefits of antiviral prophylaxis and/or vaccination.

In conclusion, new insights into the immunological mechanisms underlying allorecognition may have important clinical implications. The role of the thymus is to delete $\mathrm{T}$ cells recognizing self-peptides on autologous HLA molecules; however, many $\mathrm{T}$ cells recognizing selfpeptides presented on allogeneic HLA molecules are not deleted from the $\mathrm{T}$ cell repertoire. These thymically educated $\mathrm{T}$ cells enter the circulation, are commonly and specifically allo-HLA reactive, and are activated by viral infection or vaccination to become alloreactive memory $\mathrm{T}$ cells which are a major barrier to successful tolerance induction (Byrne et al. 1988; Adams et al. 2003; Brook et al. 2006). Antiviral prophylaxis combined with specific targeting of these alloreactive memory $\mathrm{T}$ cells using immunosuppressive drugs, monoclonal antibodies, and/or donor-specific Tregs could also provide a platform for the induction of donor-specific transplantation tolerance.

Open Access This article is distributed under the terms of the Creative Commons Attribution Noncommercial License which permits any noncommercial use, distribution, and reproduction in any medium, provided the original author(s) and source are credited.

\section{References}

Adams A, Williams M, Jones T et al (2003) Heterologous immunity provides a potent barrier to transplantation tolerance. $\mathrm{J}$ Clin Invest 111:1887-1895

Alberu J, Morales-Buenrostro L, de Leo C et al (2007) A nonallogeneic stimulus triggers the production of de-novo HLA antibodies in healthy adults. Transpl Immunol 18:166-171

Amir A, D'Orsogna L, Roelen D et al (2010) Allo-HLA reactivity of viral specific memory $\mathrm{T}$ cells is common. Blood 115:3146-3157

Anonymous (2004) Guidelines for vaccination of solid organ transplant candidates and recipients. Am J Transplant, 4 (Suppl 10): 160-163

Archbold J, Macdonald W, Miles J et al (2006) Alloreactivity between disparate cognate and allogeneic pMHC-I complexes is the result of highly focused, peptide-dependent structural mimicry. J Biol Chem 281:34324-34332

Argaet V, Schimdt C, Burrows S et al (1994) Dominant selection of an invariant $\mathrm{T}$ cell antigen receptor in response to persistent infection by Epstein-Barr virus. J Exp Med 180:2335-2340

Bankovich A, Garcia K (2003) Not just any T cell receptor will do. Immunity $18: 7-11$

Borbulevych O, Piepenbrink K, Gloor B et al (2009) T cell receptor cross-reactivity directed by antigen-dependent tuning of peptideMHC molecular flexibility. Immunity 31:885-896
Borst J, de Vries E, Spits H, de Vries J, Boylston A, Matthews E (1987) Complexity of $\mathrm{T}$ cell receptor recognition sites for defined alloantigens. J Immunol 139:1952-1959

Brehm M, Daniels K, Priyadharshini B et al (2010) Allografts stimulate cross-reactive virus-specific memory CD8 T cells with private specificity. Am J Transplant 10:1738-1748

Brook M, Wood K, Jones N (2006) The impact of memory T cells on rejection and the induction of tolerance. Transplantation 82:1-9

Burrows S, Khanna R, Burrows J, Moss D (1994) An alloresponse in humans is dominated by cytotoxic T lymphocytes (CTL) crossreactive with a single Epstein-Barr virus CTL epitope: implications for graft-vs-host disease. J Exp Med 179:1155-1161

Burrows S, Silins S, Moss D et al (1995) T cell receptor repertoire for a viral epitope in humans is diversified by tolerance to a background major histocompatibility antigen. J Exp Med 182:1703-1715

Burrows S, Silins S, Khanna R et al (1997) Cross-reactive memory T cells for Epstein-Barr virus augment the alloresponse to common human leukocyte antigens: degenerate recognition of major histocompatibility complex-bound peptide by $\mathrm{T}$ cells and its role in alloreactivity. Eur J Immunol 27:1726-1736

Burrows S, Khanna R, Silins S, Moss D (1999) The influence of antiviral T-cell responses on the alloreactive repertoire. Immunol Today 20:203-207

Byrne J, Butler J, Cooper M (1988) Differential activation requirements for virgin and memory T cells. J Immunol 141:3249-3257

Cai J, Terasaki P, Anderson N et al (2009) Intact HLA not beta2m-free heavy chain-specific HLA class I antibodies are predictive of graft failure. Transplantation 88:226-230

Colf L, Bankovich A, Hanick N et al (2007) How a single T cell receptor recognizes both self and foreign MHC. Cell 129:135-146

D'Orsogna L, Amir A, Zoet Y et al (2009) New tools to monitor the impact of viral infection on the alloreactive T-cell repertoire. Tissue Antigens 74:290-297

D’Orsogna L, Roelen D, Doxiadis I, Claas F (2010) Alloreactivity from human viral specific memory T-cells. Transpl Immunol 23:149-155

D’Orsogna L, Roelen D, van der Meer-Prins E et al (2011a) Tissue specificity of cross-reactive allogeneic responses by EBV EBNA3A specific memory T cells. Transplantation 91:494-500

D’Orsogna L, Roelen D, Doxiadis I, Claas F (2011b) Screening of viral specific T-cell lines for HLA alloreactivity prior to adoptive immunotherapy may prevent GvHD. Transpl Immunol 24:141

D’Orsogna L, van Besouw N, van der Meer-Prins E et al (2011c) Vaccine induced allo-HLA reactive memory $\mathrm{T}$ cells in a kidney transplantation candidate. Transplantation 91:645-651

Danziger-Isakov L, Cherkassky L, Siegel H et al (2010) Effects of influenza immunization on humoral and cellular alloreactivity in humans. Transplantation 89:838-844

Deckers J, Boonstra J, van der Kooij S, Daha M, van der Woude F (1997) Tissue-specific characteristics of cytotoxic graft-infiltrating T cells during renal allograft rejection. Transplantation 64:178-181

Deckers J, Daha M, van der Kooij S, van der Woude F (1998) Epithelial and endothelial-cell specificity of renal graft infiltrating T cells. Clin Transplant 12:285-291

El-Awar N, Terasaki P, Nguyen A et al (2008) Epitopes of HLA antibodies found in sera of normal healthy males and cord blood. Clin Transpl 199-214

Ely L, Burrows S, Purcell A et al (2008) T-cells behaving badly: structural insights into alloreactivity and autoimmunity. Curr Opin Immunol 20:575-580

Feng G, Chan T, Wood K, Bushell A (2009) Donor reactive regulatory T cells. Curr Opin Organ Transplant 14:432-438

Fleischhauer K, Avila D, Vilbois F et al (1994) Characterization of natural peptide ligands for HLA-B*4402 and $-B^{*} 4403$ : implications for peptide involvement in allorecognition of a single amino 
acid change in the HLA-B44 heavy chain. Tissue Antigens 44:311-317

Flynn K, Mullbacher A (1996) Memory alloreactive cytotoxic T cells do not require costimulation for activation in vitro. Immunol Cell Biol 74:413-420

Francis R, Feng G, Tha-In T et al (2011) Induction of transplantation tolerance converts potential effector $\mathrm{T}$ cells into graft-protective regulatory T cells. Eur J Immunol 41:726-738

Gaston J, Rickinson A, Epstein M (1983) Cross-reactivity of selfHLA-restricted Epstein-Barr virus-specific cytotoxic T lymphocytes for allo-HLA determinants. J Exp Med 158:1804-1821

Golshayan D, Wyss J, Buckland M et al (2010) Differential role of naïve and memory $\mathrm{CD} 4+\mathrm{T}$-cell subsets in primary alloresponses. Am J Transplant 10:1749-1759

Gras S, Burrows S, Kjer-Nielsen L et al (2009) The shaping of T cell receptor recognition by self-tolerance. Immunity 30:193-203

Gray D, Matzinger P (1991) T cell memory is short lived in the absence of antigen. J Exp Med 174:969-974

Greisemer A, Sorenson E, Hardy M (2010) The role of the thymus in tolerance. Transplantation 90:465-474

Hamann D, Baars P, Rep M et al (1997) Phenotypic and functional separation of memory and effector human CD8+ T cells. J Exp Med 186:1407-1418

Heath W, Sherman L (1991) Cell-type-specific recognition of allogeneic cells by alloreactive cytotoxic T cells: a consequence of peptide-dependent allorecognition. Eur J Immunol 21:153-159

Herman J, Jongeneel V, Kuznetsov D, Coulie P (1999) Differences in the recognition by CTL of peptides presented by the HLA$B * 4402$ and the HLA-B*4403 molecules which differ by a single amino acid. Tissue Antigens 53:111-121

Jiang S, Golshayan D, Tsang J et al (2006) In vitro expanded alloantigen-specific CD4+CD25+ regulatory $\mathrm{T}$ cell treatment for the induction of donor-specific transplantation tolerance. Int Immunopharmacol 6:1879-1882

Katerinis I, Hadaya K, Duquesnoy R et al (2011) De novo anti-HLA antibody after pandemic H1N1 and seasonal influenza immunization in kidney transplant recipients. Am J Transplant 11:1727-1733

Kleim V, Fricke L, Wollbrink T et al (2008) Improvement in long-term renal graft survival due to CMV prophylaxis with oral ganciclovir: results of a randomized clinical trial. Am J Transplant 8:975-983

Macdonald W, Chen Z, Gras S et al (2009) T cell recognition via molecular mimicry. Immunity 31:897-908

Macedo C, Orkis A, Popescu I et al (2009) Contribution of naïve and memory T-cell populations to the human alloimmune response. Am J Transplant 9:2057-2066

Marrack P, Kappler J (1988) T cells can distinguish between allogeneic major histocompatibility complex products on different cell types. Nature 332:840-843

Mason D (1998) A very high level of cross-reactivity is an essential feature of the T-cell receptor. Immunol Today 19:428-429

Melenhorst J, Leen A, Bollard C, Quigley M, Price D, Rooney C et al (2010) Allogeneic virus-specific T cells with HLA alloreactivity do not produce GVHD in human subjects. Blood 116:4700-4702

Mifsud N, Nguyen T, Tait B, Kotsimbos T (2010) Quantitative and functional diversity of cross-reactive EBV-specific CD8+ T cells in a longitudinal study cohort of lung transplant recipients. Transplantation 90:1439-1449
Miltenburg A, Meijer-Paape M, Daha M et al (1989) Donor-specific lysis of human proximal tubular epithelial cells by renal allograftinfiltrating lymphocytes. Transplantation 48:296-302

Molina I, Huber B (1990) The expression of tissue-specific self-peptide is required for allorecognition. J Immunol 144:2082-2088

Morales-Buenrostro L, Terasaki P, Marino-Vazquez L et al (2008) "Natural" human leukocyte antigen antibodies found in nonalloimmunized healthy males. Transplantation 86:1111-1115

Nagahama K, Nishimura E, Sakaguchi S (2007) Induction of tolerance by adoptive transfer of Treg cells. Methods Mol Biol 380:431-442

Pearl J, Parris J, Hale D, Hoffman S, Bernstein W, McCoy K et al (2005) Immunocompetent T-cells with a memory-like phenotype are the dominant cell type following antibody-mediated T-cell depletion. Am J Transplant 5:465-474

Rist M, Smith C, Beil M et al (2009) Cross-recognition of HLA-DR4 alloantigen by virus-specific CD8+ T-cells: a new paradigm for self/non-self recognition. Blood 114:2244-2253

Schild H, Rotzschke O, Kalbacher H, Rammensee H (1990) Limit of $\mathrm{T}$ cell tolerance to self-proteins by peptide presentation. Science 247:1587-1589

Selin L, Brehm M (2007) Frontiers in nephrology: heterologous immunity, T cell cross-reactivity and alloreactivity. J Am Soc Nephrol 18:2268-2277

Umetsu D, Yunis E, Matsui Y, Jabara H, Geha R (1985) HLA-DR4associated alloreactivity of an HLA-DR3-restricted tetanus toxoid-specific T-cell clone: inhibition of both reactivities by an alloantiserum. Eur J Immunol 15:356-361

van der Woude F, Daha M, Miltenburg A et al (1990) Renal allograftinfiltrated lymphocytes and proximal tubular cells: further analysis of donor-specific lysis. Hum Immunol 28:186-192

Venturi V, Price D, Douek D, Davenport M (2008) The molecular basis for public T-cell responses? Nat Rev Immunol 8:231-238

Wang T, Chen L, Ahmed E et al (2008) Prevention of allograft tolerance by bacterial infection with Listeria monocytogenes. J Immunol 180:5991-5999

Webb S, Sprent J (1986) T cells with multiple specificities. Int Rev Immunol 1:151

Welsh R, Selin L (2002) No one is naïve: the significance of heterologous T-cell immunity. Nat Rev Immunol 2:417-426

Welsh R, Markees T, Woda B et al (2000) Virus-induced abrogation of transplantation tolerance induced by donor-specific transfusion and anti-CD154 antibody. J Virol 74:2210-2218

Whitelegg A, Barber L (2004) The structural basis of T-cell allorecognition. Tissue Antigens 63:101-108

Yang H, Welsh R (1986) Induction of alloreactive cytotoxic T cells by acute virus infection of mice. J Immunol 136:1186-1193

Yard B, Claas F, Paape M et al (1994) Recognition of a tissue-specific polymorphism by graft infiltrating T-cell clones isolated from a renal allograft with acute rejection. Nephrol Dial Transplant 9:805-810

Yin Y, Marluzza R (2009) The multiple mechanisms of T cell receptor cross-reactivity. Immunity 31:849-851

Yi-qun Z, van Neervan J, Kasran A, de Boer M, Ceuppens J (1996) Differential requirements for co-stimulatory signals from B7 family members by resting versus recently activated memory $\mathrm{T}$ cells towards soluble recall antigens. Int Immunol 8:37-44

Zerrahn J, Held W, Raulet D (1997) The MHC reactivity of the T cell repertoire prior to positive and negative selection. Cell 88:627-636 